\title{
Blood eosinophil count: a biomarker of an important treatable trait in patients with airway disease
}

\author{
Ian D. Pavord ${ }^{1}$ and Alvar Agusti ${ }^{2}$ \\ Affiliations: ${ }^{1}$ Respiratory Medicine Unit, Nuffield Department of Medicine, NDM Research Building, Old Road \\ Campus, University of Oxford, Oxford, UK. ${ }^{2}$ Respiratory Institute, Hospital Clinic, IDIBAPS, Univ. Barcelona \\ and CIBER Enfermedades Respiratorias (CIBERES), Spain.
}

Correspondence: Alvar Agusti, Respiratory Institute, Hospital Clinic, C/ Villarroel 170 (Escala 3, Planta 5), 08036 Barcelona, Spain. E-mail: Alvar.Agusti@clinic.ub.es

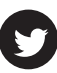

@ERSpublications

Blood eosinophil count can identify eosinophilic airway inflammation, a "treatable trait" of COPD http://ow.ly/Xn098

Inhaled corticosteroids (ICS) are widely used to treat patients with chronic obstructive pulmonary disease (COPD). Their main impact is to reduce the risk of exacerbations; in contrast to long-acting bronchodilators their effects on symptoms and lung function are small and insufficient to use as a guide of treatment efficacy. As a result, ICS are applied in a "risk-directed" fashion, with treatment directed at patients deemed to be at risk of exacerbations because of a past history of events, and/or poor lung function [1]. A better, more precise strategy would be to apply treatment in a targeted fashion according to a biomarker indicating the likelihood that corticosteroid treatment will have a positive effect on the outcome of interest (i.e. reduction in exacerbations or decline in lung function).

Interest in better targeting of ICS treatment has increased recently for three reasons [2]. First, there has been increasing recognition of the potentially adverse effects of ICS treatment. Pneumonia risk increases as a function of ICS dose and treatment duration [3] and there are many other potential adverse effects, including increased risk of tuberculosis and nontuberculosis mycobacterial infection [4]. Second, ICS use, particular high dose potent ICS use, has increased substantially in the last 10 years and much of this use is outside current guidelines [5]. Withdrawing ICS may be difficult to do in a condition when the primary goal of treatment is to reduce future events, and trials of ICS withdrawal have had a limited impact [6]. And, finally, we have other potentially attractive treatment options for patients with symptomatic COPD, notably the combination of long-acting $\beta_{2}$-agonists (LABA) and long acting anti-muscarinic agents (LAMA). These agents have generally greater effects on symptoms and lung function than ICS/LABA and might have a similar effect on exacerbation frequency [7]. However, their use has been limited by the lack of satisfactory information on whom should we switch from ICS/LABA to LABA/LAMA and how we should do this.

In order to move from a risk directed to a biomarker directed ICS treatment strategy, the biomarker should indicate a corticosteroid responsive element on the causal pathway of the aspect of the condition we are attempting to modify. The induced sputum eosinophil count, a biomarker of eosinophilic airway inflammation, is an established candidate biomarker. Proof-of-concept studies have shown a consistent and compelling relationship between a raised induced sputum eosinophil count and the short-term response to oral $[8,9]$ and inhaled [10] corticosteroids. This is also the case for long-term management, since management guided by the sputum eosinophil count results in better outcomes than guideline-based management [11, 12]. However, there are other requirements for a biomarkers to be clinically useful, including being readily accessible in ordinary clinical practice, applicable in all patients, and to have well understood measurement characteristics [13]. Here the sputum eosinophil count has problems. Few centres outside specialist units offer this test in routine clinical practice and even in those that do, the technique is labour intensive and limited by a failure rate of $10-20 \%$ [14].

Received: Jan 092016 | Accepted: Jan 122016

Conflict of interest: Disclosures can be found alongside the online version of this article at erj.ersjournals.com

Copyright @ERS 2016 
An important recent advance has been the recognition that the peripheral blood eosinophil count has potential utility as a marker of eosinophilic airway inflammation and likely corticosteroid response. BAFADHEL et al. [15] showed that of the many potential biomarkers assessed, the blood eosinophil count was the stand out biomarker of exacerbations associated with a raised sputum eosinophil count. A count of $<2 \%$ (equivalent to a total eosinophil count of $0.15-0.2 \times 10^{9}$ per L) at the time of an exacerbation had a high sensitivity and thus potential as a rule out test. Similar findings have been reported for patients with stable COPD studied as part of the ECLIPSE cohort [16]. The validity of a count in this range as a marker of abnormal eosinophilic inflammation is supported by a study showing it represents the point above which a response to mepolizumab (a specific inhibitor of eosinophilic inflammation) becomes apparent in patients with severe asthma [17], and the upper limit of the normal range of blood eosinophil count in normal, non-atopic subjects [18].

A paper published in this issue of the European Respiratory Journal (ERJ) adds new and important information on the relationship between the blood and sputum eosinophil count in patients with COPD. SCHLEICH et al. [19] report an area under the receiver operating curve of 0.75 in 155 patients with COPD of varying severity and treatment requirements for blood eosinophils to predict sputum eosinophilia. Although the sensitivity and specificity of a cut point of $<2 \%$, or the equivalent total eosinophil count, is not reported, it is likely not dissimilar to previous reports, and use of inhaled corticosteroids did not seem to confound the relationship. One problem with use of the blood eosinophil count is that it provides no information on the site of inflammation and it is therefore likely to lack specificity as a biomarker of eosinophilic airway inflammation. Including a more airway specific biomarker such as exhaled nitric oxide ( $F$ eNO) might overcome this problem, in which case a composite of blood eosinophil count and FeNO would provide better predictive value, as has been shown in asthma [20]. Yet, SchleICH et al. [19] found that this was not the case in patients with COPD, possibly reflecting the variable effect of smoking and airway infection on FeNO in patients with more complex airway disease.

Studies such as this are limited as they only provide information on the relationship between two surrogate measures (blood and sputum eosinophil counts), when the main interest is whether the biomarker provides clinically important predictive value. How does the blood eosinophil count fare as a predictor of the response to ICS of clinically important events such as exacerbations, mortality or decline in forced expiratory volume in $1 \mathrm{~s}(\mathrm{FEV} 1)$ ? Three recent re-analyses of large trials of ICS-containing treatments in COPD have shown a compelling and close relationship between the blood eosinophil count and the risk of exacerbations in patients with COPD. More importantly, they have also shown that the reduced risk of exacerbations seen with the addition of an ICS to a LABA [21, 22] or with ICS/LABA versus LAMA [23] is absent or minimal in patients with a blood eosinophil count $<2 \%$ but increases progressively above this level. In contrast, treatments not expected to modulate eosinophilic airway inflammation such as LAMA [23] or the p38 mitogen-activated protein kinase inhibitor losmapimod [24] work best in patients with a blood eosinophil count $<2 \%$. The blood eosinophil count also has utility as a marker of the response to oral corticosteroid therapy given to hasten recovery at the time of an exacerbation. All of the benefits of treatment occurs in subjects with a blood eosinophil count $\geqslant 2 \%$ [25] and there is evidence of net harm in patients with a count $<2 \%$ [26].

Exacerbations are not the only outcome of interest in COPD. Others like mortality, lung function decline over time and health-status are also of clinical relevance and, interestingly, the blood eosinophil count appears related to all. Hospers et al. [27] showed that a blood eosinophil count $>0.275 \times 10^{9}$ per L is associated with a $40 \%$ increase in mortality in a community population followed up over 30 years, and a 4.8 fold increased risk of death from COPD in patients with a history of exacerbations [28]. The effect of ICS either alone or in combination with LABA on mortality in COPD seen in the Towards a Revolution in COPD Health study (TORCH) was small and of borderline statistical significance [29]. Might the signal be greater in patients stratified by blood eosinophil count? Unfortunately, we have no way of testing this important question, as the blood eosinophil count was not measured in the TORCH study. Inclusion of this variable as a pre-specified stratification variable is clearly important for future studies.

The reported effect of ICS on FEV1 decline is small and inconsistent. The best data comes from the TORCH study, which showed that inhaled fluticasone dipropionate either alone or in combination with Salmeterol reduced the decline in FEV1 over 3 years by $20-30 \%$ [30]. Other smaller studies have shown variable findings but some did measure the blood eosinophil count at baseline, providing the opportunity to perform a post hoc stratified analysis. The first such analysis is also published in this issue of the journal. BARNES et al. [31] re-analysed the Inhaled Steroids in Obstructive Lung Disease in Europe (ISOLDE) study [32], a 20-year-old placebo-controlled randomised study of 751 patients with moderate-severe COPD which showed no effect of inhaled fluticasone dipropionate $500 \mu \mathrm{g}$ twice daily on FEV1 decline over 3 years. Interpretation of their re-analysis is problematic as there were significant numbers of drop outs, particularly before randomisation and in the group randomised to placebo. Moreover, the timing of the blood eosinophil count was not standardised and, in 43 subjects, was during or shortly after a course of prednisolone. Despite these limitations two relatively strong conclusions can be drawn: first, in the placebo treated group the rate of FEV1 
decline was greater in the $30 \%$ of patients with an eosinophil count $\geqslant 2 \%$ than those with a count $<2 \%(74.5$ versus $51.3 \mathrm{~mL} \cdot$ year $^{-1}$ ); and second, in the ICS treated patients the excess decline was reduced substantially in the $\geqslant 2 \%$ group (to $40.6 \mathrm{~mL} \cdot$ year $^{-1}$ ) but not in the $<2 \%$ group $\left(54.2 \mathrm{~mL} \cdot\right.$ year $^{-1}$ ). Thus it seems that one of the major aims of COPD management - to slow disease progression - is achieved very effectively with ICS treatment in an important and definable subgroup of patients. Recent findings in three large and independent cohorts, however, have clearly shown that an enhanced FEV1 decline with age is not an obligatory feature of COPD, as assumed traditionally, and that low FEV1 in early adulthood is also important in the genesis of COPD [33]. This possibility has not yet been considered in any analysis of the effects of ICS on the rate of lung function decline, nor has it been appreciated before that markers of eosinophilic airway inflammation might be associated with an increased rate of decline.

Several other aspects of BARNES et al. [31] analysis merit further discussion. In contrast to other larger studies [21-23], there was no apparent difference in the effect of ICS on exacerbation rates in patients stratified by blood eosinophils. One potential explanation is that more clear-cut eosinophil associated differences are seen when ICS are added to LABA than with the ICS monotherapy evaluated in the ISOLDE study. A more likely explanation is that the ISOLDE population was a much smaller and less responsive population because withdrawal of the most eosinophilic and potentially ICS responsive patients occurred after ICS withdrawal and before randomisation. This would also explain the lower overall eosinophil count in ISOLDE than seen in other trials. The effect of ICS on decline in quality of life as assessed by the St George's Respiratory Questionnaire was less obviously linked to the baseline blood eosinophil count than decline in FEV1. This was also the case for the short-term change in FEV1 seen in this and earlier studies $[21,22]$. Collectively these findings suggest that improvement in these measures occur via a different mechanism to that responsible for decline in lung function and the occurrence of exacerbations.

Our overall impression is that there is now a compelling body of evidence linking the blood eosinophil count to clinically important outcomes and treatment responses in patients with airway disease and that it more than deserved its epithet as Thorax's biomarker of 2013 [34]. Although much of the data is derived from post hoc analyses of existing data, the biological rationale is strong and the consistency of the main findings impressive. Clearly, more prospective work is needed but should we wait for this before changes are made? We think not. We have argued that clinicians, guideline groups and researchers have become

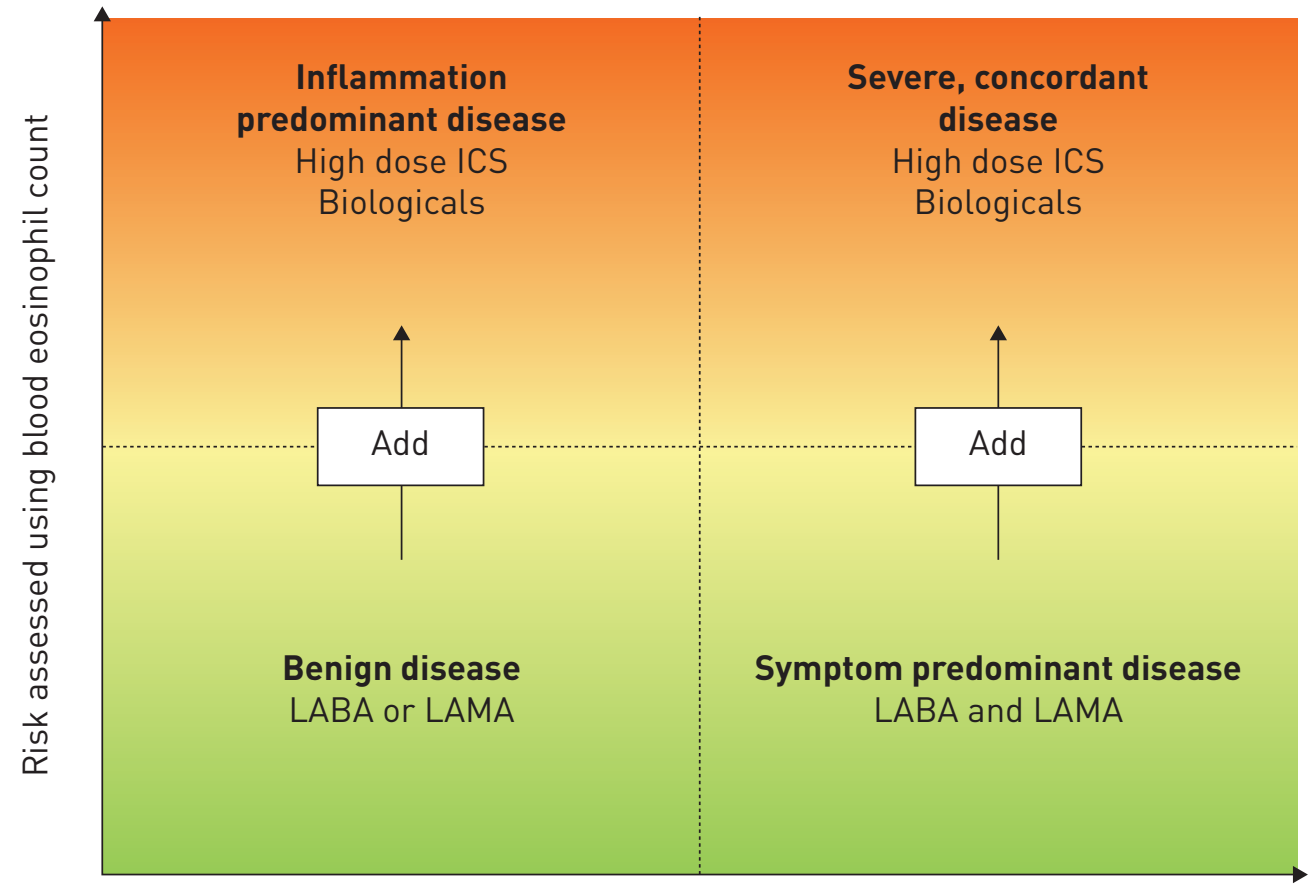

Symptoms due to airflow limitation

FIGURE 1 Proposed management of airway disease incorporating two major treatable traits: symptoms due to airflow limitation and risk assessed using the blood eosinophil count. Risk refers to future risk of exacerbation and decline in forced expiratory volume in $1 \mathrm{~s}$. Treatments with a trait specific effect are included. Rescue short-acting bronchodilators (short-acting $\beta_{2}$-agonists or short-acting muscarinic agents) could be used in all situations and patient categories, as required. ICS: inhaled corticosteroids; LABA: long-acting $\beta_{2}$-agonists; LAMA: long-acting muscarinic agonists. 
bogged down with arbitrary and unhelpful disease labels and sub-optimum management paradigms and should instead focus on identifying specific, well-defined and treatable aspects of airway disease [35].

Considering all the arguments discussed above, one of the more important "treatable traits", associated particularly with important longer-term outcomes, is eosinophilic airway inflammation, and, in the blood eosinophil count, we have a means of identifying it. Moreover, with a relatively minor change in the Global Initiative for Chronic Obstructive Lung Disease treatment pathway [1], we could integrate this new biomarker relatively simply, offering a treatment paradigm that is not only applicable in non-specialist settings but results in more personalised and potentially more effective treatment (figure 1).

\section{References}

1 Vestbo J, Hurd SS, Agusti AG, et al. Global strategy for the diagnosis, management, and prevention of chronic obstructive pulmonary disease: GOLD executive summary. Am J Respir Crit Care Med 2013; 187: 347-365.

2 Agusti A, Fabbri LM. Inhaled steroids in COPD: when should they be used? Lancet Respir Med 2014; 2: 869-871.

3 Suissa S, Patenaude V, Lapi F, et al. Inhaled corticosteroids in COPD and the risk of serious pneumonia. Thorax 2013; 68: 1029-1036.

4 Sabroe I, Postma D, Heijink I, et al. The yin and the yang of immunosuppression with inhaled corticosteroids. Thorax 2013: 1085-1087.

5 Brusselle G, Price D, Gruffydd-Jones K, et al. The inevitable drift to triple therapy in COPD: an analysis of prescribing pathways in the UK. Int J Chron Obstruct Pulmon Dis 2015; 10: 2207-2217.

6 Magnussen H, Disse B, Rodriguez-Roisin R, et al. Withdrawal of inhaled glucocorticoids and exacerbations of COPD. N Engl J Med 2014; 371: 1285-1294.

7 Singh D. New combination bronchodilators for chronic obstructive pulmonary disease: current evidence and future perspectives. Br J Clin Pharmacol 2015; 79: 695-708.

8 Pizzichini E, Pizzichini MM, Gibson P, et al. Sputum eosinophilia predicts benefit from prednisone in smokers with chronic obstructive bronchitis. Am J Respir Crit Care Med 1998; 158: 1511-1517.

9 Brightling CE, Monteiro W, Ward R, et al. Sputum eosinophilia and short-term response to prednisolone in chronic obstructive pulmonary disease: a randomised controlled trial. Lancet 2000; 356: 1480-1485.

10 Brightling CE, McKenna S, Hargadon B, et al. Sputum eosinophilia and the short term response to inhaled mometasone in chronic obstructive pulmonary disease. Thorax 2005; 60: 193-198.

11 Siva R, Green RH, Brightling CE, et al. Eosinophilic airway inflammation and exacerbations of COPD: a randomised controlled trial. Eur Respir J 2007; 29: 906-913.

12 McDonald VM, Higgins I, Wood LG, et al. Multidimensional assessment and tailored interventions for COPD: respiratory utopia or common sense? Thorax 2013; 68: 691-694.

13 Jones PW, Agusti AG. Outcomes and markers in the assessment of chronic obstructive pulmonary disease. Eur Respir J 2006; 27: 822-832.

14 Pavord ID, Pizzichini MM, Pizzichini E, et al. The use of induced sputum to investigate airway inflammation. Thorax 1997; 52: 498-501.

15 Bafadhel M, McKenna S, Terry S, et al. Acute exacerbations of chronic obstructive pulmonary disease: identification of biologic clusters and their biomarkers. Am J Respir Crit Care Med 2011; 184: 662-671.

16 Singh D, Kolsum U, Brightling CE, et al. Eosinophilic inflammation in COPD: prevalence and clinical characteristics. Eur Respir J 2014; 44: 1697-1700.

17 Pavord ID, Korn S, Howarth P, et al. Mepolizumab for severe eosinophilic asthma (DREAM): a multicentre, double-blind, placebo-controlled trial. Lancet 2012; 380: 651-659.

18 Felarca A, Lowell F. The total eosinophil count in a non-atopic population. J Allergy 1967; 40: 4.

19 Schleich F, Corhay J-L, Louis R. Blood eosinophil count to predict bronchial eosinophilic inflammation in COPD. Eur Respir J 2016; 47: 1562-1564.

20 Malinovschi A, Fonseca JA, Jacinto T, et al. Exhaled nitric oxide levels and blood eosinophil counts independently associate with wheeze and asthma events in National Health and Nutrition Examination Survey subjects. J Allergy Clin Immunol 2013; 132: 821-827.

21 Pascoe S, Locantore N, Dransfield MT, et al. Blood eosinophil counts, exacerbations, and response to the addition of inhaled fluticasone furoate to vilanterol in patients with chronic obstructive pulmonary disease: a secondary analysis of data from two parallel randomised controlled trials. Lancet Respir Med 2015; 3: 435-442.

22 Siddiqui SH, Guasconi A, Vestbo J, et al. Blood Eosinophils: A Biomarker of Response to Extrafine Beclomethasone/ Formoterol in Chronic Obstructive Pulmonary Disease. Am J Respir Crit Care Med 2015; 192: 523-525.

23 Pavord ID, Lettis S, Locantore N, et al. Blood eosinophils and inhaled corticosteroid/long-acting $\beta-2$ agonist efficacy in COPD. Thorax 2016; 71: 118-125.

24 Marks-Konczalik J, Costa M, Robertson J, et al. A post-hoc subgroup analysis of data from a six month clinical trial comparing the efficacy and safety of losmapimod in moderate-severe COPD patients with $\leqslant 2 \%$ and $>2 \%$ blood eosinophils. Respir Med 2015; 109: 860-869.

25 Bafadhel M, Davies L, Calverley PM, et al. Blood eosinophil guided prednisolone therapy for exacerbations of COPD: a further analysis. Eur Respir J 2014: 789-791.

26 Bafadhel M, McKenna S, Terry S, et al. Blood eosinophils to direct corticosteroid treatment of exacerbations of chronic obstructive pulmonary disease: a randomized placebo-controlled trial. Am J Respir Crit Care Med 2012; 186: $48-55$

27 Hospers JJ, Schouten JP, Weiss ST, et al. Eosinophilia is associated with increased all-cause mortality after a follow-up of 30 years in a general population sample. Epidemiology 2000; 11: 261-268.

28 Hospers JJ, Schouten JP, Weiss ST, et al. Asthma attacks with eosinophilia predict mortality from chronic obstructive pulmonary disease in a general population sample. Am J Respir Crit Care Med 1999; 160: 1869-1874.

29 Calverley PM, Anderson JA, Celli B, et al. Salmeterol and fluticasone propionate and survival in chronic obstructive pulmonary disease. N Engl J Med 2007; 356: 775-789. 
30 Celli BR, Thomas NE, Anderson JA, et al. Effect of pharmacotherapy on rate of decline of lung function in chronic obstructive pulmonary disease: results from the TORCH study. Am J Respir Crit Care Med 2008; 178: 332-338.

31 Barnes NC, Sharma R, Lettis S, et al. Blood eosinophils as a marker of response to inhaled corticosteroids in COPD. Eur Respir J 2016; 47: 1374-1382.

32 Burge PS, Calverley PM, Jones PW, et al. Randomised, double blind, placebo controlled study of fluticasone propionate in patients with moderate to severe chronic obstructive pulmonary disease: the ISOLDE trial. BMJ 2000; 320: 1297-1303.

33 Lange P, Celli B, Agusti A, et al. Lung-function trajectories leading to chronic obstructive pulmonary disease. N Engl J Med 2015; 373: 111-122.

34 Bush A, Pavord I. Year in review 2013: paediatric and adult clinical studies. Thorax 2014; 69: 309-311.

35 Agusti A, Bel E, Thomas M, et al. Treatable traits: toward precision medicine of chronic airway diseases. Eur Respir J 2016; 47: 410-419. 